\section{Enhancement of Warfarin Anticoagulant Reaction in Patients with Repeated Oral Tolvaptan Administration}

\author{
Masayuki Saito, ${ }^{a, b}$ Masayoshi Ajioka, ${ }^{c}$ \\ Takahiro Iwao, ${ }^{b}$ and Tadashi Suzuki* ${ }^{*}, b$ \\ ${ }^{a}$ Department of Pharmacy, Tosei General Hospital; 160 Nishi- \\ oiwake-cho, Seto, Aichi 489-8642, Japan: ${ }^{b}$ Department of \\ Clinical Pharmacy, Graduate School of Pharmaceutical Sciences, \\ Nagoya City University; 3-1 Tanabe-dori, Mizuho-ku, Nagoya \\ 467-8603, Japan: and ${ }^{c}$ Department of Cardiovascular Medicine, \\ Tosei General Hospital; 160 Nishi-oiwake-cho, Seto, Aichi 489- \\ 8642, Japan. \\ Received December 15, 2017; accepted April 30, 2018; advance \\ publication released online May 11, 2018 \\ The pharmacokinetics and pharmacodynamics of warfarin \\ remained unaffected by tolvaptan during clinical trials. How- \\ ever, tolvaptan prolonged the prothrombin time-international \\ normalized ratio (PT-INR) level of patients with cardiovas- \\ cular disease taking warfarin. Tolvaptan was prescribed to \\ 576 patients from December 2010 to December 2015. Of these \\ patients, 37 underwent anticoagulant therapy. We investigated \\ PT-INR fluctuation immediately before tolvaptan therapy \\ was initiated. PT-INR remained unchanged in the control \\ group and in groups administered with less than $7.5 \mathrm{mg} / \mathrm{d}$ \\ tolvaptan, whereas it was significantly increased $(p=0.03)$ in \\ the group administered with more than $7.5 \mathrm{mg} / \mathrm{d}$ tolvaptan. \\ This result indicates the possibility that tolvaptan affects \\ the pharmacodynamics of warfarin in vivo. However, further \\ research is necessary to clarify the mechanism of this phe- \\ nomenon.
}

Key words pharmacist; phamacodynamics; tolvaptan; warfarin

Tolvaptan is a diuretic medication that is used for the treatment of heart failure, hepatic cirrhosis, and autosomal dominant polycystic kidney disease (ADPKD). This drug acts as an antagonist of the vasopressin V2 receptor and discharges the water from the interior of the body.

Some pharmacokinetic and pharmacodynamic studies, including those on the influences of multiple drugs, have been conducted, ${ }^{1)}$ and particularly tolvaptan has been reported to not interact with warfarin. ${ }^{2)}$ However, we observed an interaction between tolvaptan and warfarin from vital signs and laboratory data of patients with cardiovascular disease. Tolvaptan increased the prothrombin time-international normalized ratio (PT-INR) level of patients taking warfarin. With the aims of preventing side effects, conducting proper drug treatment, and informing clinical staff, we verified the pharmacodynamic interaction of tolvaptan in patients repeatedly administered with oral warfarin.

\section{PATIENTS AND METHODS}

Definition of Subjects and Control Patients Tolvaptan was prescribed to 576 patients from December 2010 to December 2015 in the Tosei General Hospital, of which 37 (19 females) underwent warfarin therapy. We investigated
PT-INR fluctuation immediately before tolvaptan therapy was initiated. PT-INR used in this study is the corrected value in the index that compared prothrombin activity measured by the reagent from each company with the activity measured by the international standard. Tolvaptan dosage and frequency were at the discretion of the physicians.

In this study, we excluded 539 patients (408 patients without warfarin intake; in 47 patients, PT-INR values were not measured before and after tolvaptan intake; 35 patients began to take CYP inhibitors and/or inducers, which could influence the pharmacokinetics of warfarin; in 26 patients, warfarin dosages were changed; four patients had compliance problems; three patients had fever, diarrhea, or a change in daily diet; in three patients, tolvaptan administration was terminated; in 12 patients, warfarin was changed to the other anticoagulant drugs; and one patient progressed to a severe disease state). PT-INR standard values were measured the day before tolvaptan administration, and the largest values were recorded after the administration. PT-INR values during hospitalization were used. In addition, all patients in the study were hospitalized and administered tolvaptan daily. The control group comprised patients who were admitted into our hospital from January 2008 to March 2014 under the same conditions as those of the subject group. In the control groups, PT-INR values on hospitalization were used as the standard, and maximum values during hospitalization were similarly recorded. There was no significant difference in age, sex, comorbidity, and warfarin dosage between the two groups.

Ethics This research was conducted according to the medical ethics of our institution.

Statistical Analyses We used StatView 5.0 (SAS Institute Inc., Cary, NC, U.S.A.) and SPSS (IBM, Armonk, NY, U.S.A.) for statistical analyses. We used ANOVA to evaluate differences of warfarin dosage among groups of each dosage of tolvaptan. We analyzed the relationship between changes of PT-INR values and tolvaptan dosages using paired $t$-test. Measured values were presented as median \pm confidence interval. $p<0.05$ was considered statistically significant.

\section{RESULTS}

We divided the patients into two groups based on the difference of tolvaptan dosage (below $7.5 \mathrm{mg} / \mathrm{d}$, or over $7.5 \mathrm{mg} / \mathrm{d}$ ). Figure 1 shows that PT-INR values of the control group $(p=0.32)$ remained unchanged. Similarly, for groups administered below $7.5 \mathrm{mg} / \mathrm{d}$ tolvaptan, PT-INR fluctuation was not observed $(p=0.96)$. However, all patients taking tolvaptan had a significantly increased PT-INR value $(p=0.008)$. In addition, PT-INR level was significantly increased $(p=0.003)$ in the group taking over $7.5 \mathrm{mg} / \mathrm{d}$ tolvaptan. This result indicated the possibility that tolvaptan affects the pharmacodynamics of warfarin in vivo, suggesting dose dependence. Each warfarin dosage was $2.05 \pm 0.86$, and $2.10 \pm 0.88 \mathrm{mg} / \mathrm{d}$ in the groups administered below $7.5 \mathrm{mg} / \mathrm{d}$, and over $7.5 \mathrm{mg} / \mathrm{d}$ of tolvaptan, respectively. Statistical analysis revealed no significant differences in warfarin dosage among the groups administered with tolvaptan $(F=0.97, p=0.96)$. 

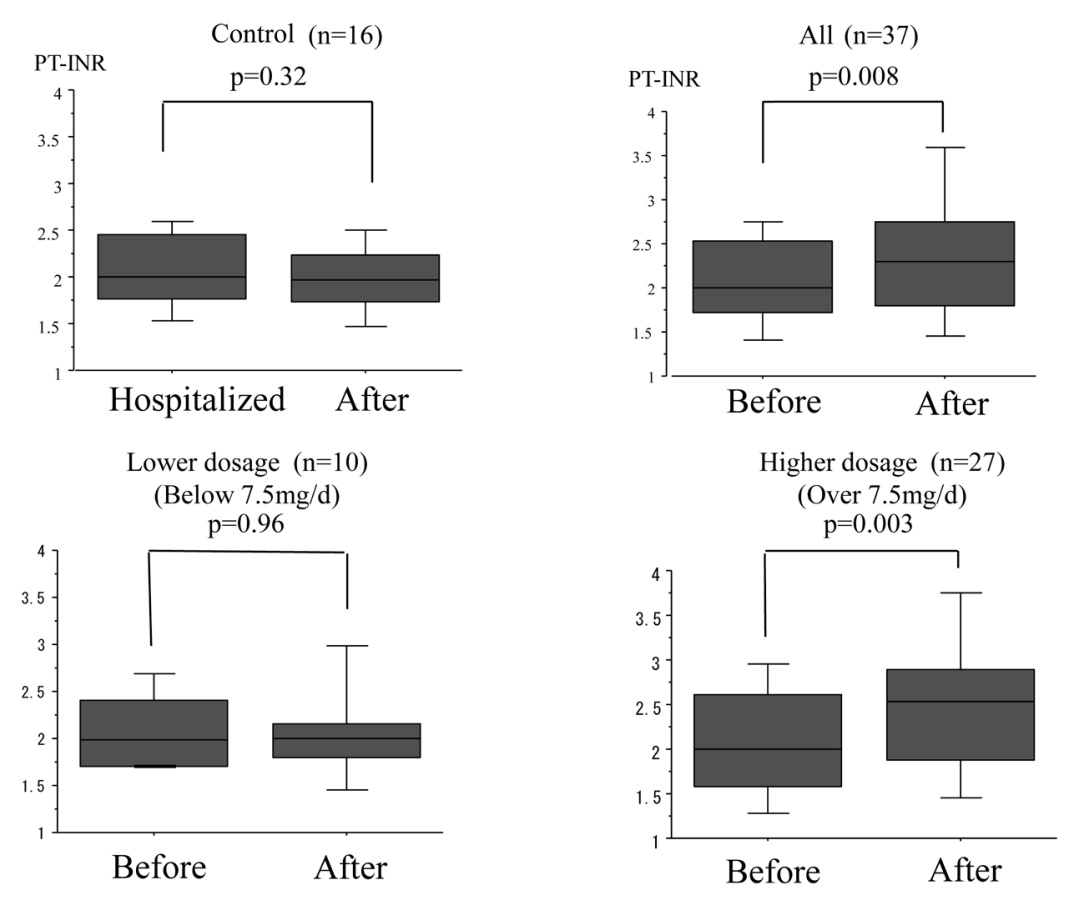

Fig. 1. PT-INR Values before and after Tolvaptan Intake

PT-INR values in the control group during hospitalization are shown. Changes in the values before and after tolvaptan intake are shown. All data are presented as median \pm confidence interval.

\section{DISCUSSION}

In this study, we observed the interaction between tolvaptan and warfarin in patients with cardiovascular disease, which suggested that tolvaptan increased PT-INR levels in patients with warfarin intake. However, we cannot eliminate the possibility that tolvaptan directly influences the change in PT-INR levels. So, we measured PT-INR values of patients with hepatic cirrhosis and ADPKD who used tolvaptan but not warfarin; however, the values were unchanged. Thus, the possibility that tolvaptan directly influences PT-INR values was low.

To date, increased PT-INR values have commonly been considered to be associated with various changes of status (such as fever, diarrhea, and diet) ${ }^{3)}$ and drug interactions. ${ }^{4)}$ However, tolvaptan has been reported to not affect the pharmacokinetics and pharmacodynamics of warfarin. ${ }^{1,2)}$ The major difference between these studies and our study is the single dose of tolvaptan and warfarin used in these compared with multiple doses in our study. The half-life of warfarin is approximately $30-40 \mathrm{~h},{ }^{5)}$ and the fraction unbound to serum proteins is responsible for the anticoagulant activity. ${ }^{6}$ Repeated administration may lead to rises of free drug concentration due to the change of protein binding rate. Thus, our results suggested that tolvaptan increases PT-INR values in patients with repeated warfarin intake.

The free blood concentration of warfarin is increased by the replacement of protein binding. ${ }^{7,8)}$ The protein binding rate of warfarin might have been changed by tolvaptan in this study because the protein binding rate of tolvaptan is high. To the best of our knowledge, no studies have reported the interaction between warfarin and tolvaptan via CYP. Therefore, to clarify the mechanisms of increased PT-INR levels in detail, further research is needed, including the pharmacokinetic analysis of warfarin.

The average days were $7.5 \pm 3.4 \mathrm{~d}$ after the administration of tolvaptan until 0.5 -point increase in PT-INR values. However, the values were not measured daily in our study. An accurate evaluation of PT-INR increases could be possible by measuring the values daily. In addition, based on the knowledge in this research, for patients with risks for an increase in PT-INR levels (such as fever, antibiotic drug use, diarrhea, and bleeding risk), there is a need to control warfarin dosage when beginning or stopping tolvaptan. In this study, the administration of warfarin was stopped in two cases, and warfarin dosage was reduced in eight cases. In these cases, PT-INR returned to within the proper range after the dosage of warfarin was decreased or its administration was stopped. We have been alerting patients after hospital discharge to be careful because PT-INR measurement interval is extended and bleeding tendency may increase. The side effects and drug interactions described in the package inserts may also be caused by continuous administration even if not by a single dose. We believe that pharmacists should take notice as a member of medical workers to support drug treatments in the medical field.

\section{CONCLUSION}

In this study, we found the possibility that tolvaptan enhances anticoagulant action in patients with repeated oral administration of warfarin. The interaction between the two drugs is not mentioned in the package inserts. Pharmacists may have to inform the possibility of increased PT-INR levels to physicians and other clinical staffs when tolvaptan is initiated. We began to check PT-INR more frequently after obtaining this knowledge, and could thereby contribute to the treatments.

When pharmacists provide drug information to physicians for supporting drug treatments, they not only judge from the information in the package inserts but also continuously ob- 
serve the actual data and status of patients, which, if not done, would cause serious side effects and adverse influences due to drug treatment.

Conflict of Interest The authors declare no conflict of interest.

\section{REFERENCES}

1) Kim SR, Hasunuma T, Sato O, Okada T, Kondo M, Azuma J. Pharmacokinetics, pharmacodynamics and safety of tolvaptan, a novel, oral, selective nonpeptide AVP V2-receptor antagonist: results of single- and multiple-dose studies in healthy Japanese male volunteers. Cardiovasc. Drugs Ther., 25 (Suppl. 1), S5-S17 (2011).

2) Shoaf SE, Mallikaarjun S. Pharmacokinetic and pharmacodynamic interaction between tolvaptan and warfarin in healthy subjects. Clin. Pharmacol. Drug Dev., 1, 67-75 (2012).

3) Karlson B, Leijd B, Hellström K. On the influence of vitamin Krich vegetables and wine on the effectiveness of warfarin treatment.
Acta Med. Scand., 220, 347-350 (1986).

4) Clark NP, Delate T, Riggs CS, Witt DM, Hylek EM, Garcia DA, Ageno W, Dentali F, Crowther MA, Warfarin-Associated Research Projects and Other Endeavors Consortium. Warfarin interactions with antibiotics in the ambulatory care setting. JAMA Intern. Med., 174, 409-416 (2014).

5) Vesell ES, Shively CA. Liquid chromatographic assay of warfarin: similarity of warfarin half-lives in human subjects. Science, $\mathbf{1 8 4}$, 466-468 (1974).

6) O'reilly RA, Aggeler PM, Leong LS. Studies on the coumarin anticoagulant drugs: a comparison of the pharmacodynamics of dicumarol and warfarin in man. Thromb. Diath. Haemorrh., 11, 1-22 (1964).

7) Sellers EM, Koch-Weser J. Displacement of warfarin from human albumin by diazoxide and ethacrynic, mefenamic, and nalidixic acids. Clin. Pharmacol. Ther., 11, 524-529 (1970).

8) Solomon HM, Schrogie JJ. The effect of various drugs on the binding of warfarin-14C to human albumin. Biochem. Pharmacol., 16, 1219-1226 (1967). 